\title{
Comparisons of MVAC and MVDC Systems in Dynamic Operation, Fault Protection and Post-Fault Restoration
}

\author{
Gen Li \\ School of Engineering \\ Cardiff University \\ Cardiff, United Kingdom \\ Lig9@cardiff.ac.uk \\ Jun Liang \\ School of Engineering \\ Cardiff University \\ Cardiff, United Kingdom \\ LiangJ1@cardiff.ac.uk
}

\author{
Lu Zhang \\ College of Information and Electrical \\ Engineering \\ China Agricultural University \\ Beijing, China \\ zhanglu1@cau.edu.cn \\ Gangui Yan \\ Key Laboratory of Modern Power System \\ Simulation and Control \& Renewable Energy \\ Technology, Ministry of Education \\ Northeast Electric Power University \\ Jilin, China \\ yangg@neepu.edu.cn
}

\author{
Tibin Joseph \\ School of Engineering \\ Cardiff University \\ Cardiff, United Kingdom \\ JosephT@cardiff.ac.uk
}

\begin{abstract}
One of the most significant obstacles preventing the large-scale application of direct-current (DC) technology in medium voltage (MV) distribution networks is their fault protection. The existing $\mathrm{AC}$ relay protection needs to be changed or redesigned to protect the future overlay MVAC and MVDC distribution networks. Therefore, a comprehensive understanding of the dynamic and fault behavior and post-fault restoration strategies of MVAC and MVDC systems are critically important. Moreover, a comparison of MVAC and MVDC systems during a fault will also contribute to designing the protection systems of hybrid MV AC/DC systems. In this paper, the challenges of protecting DC faults of MVDC systems and possible solutions are first introduced. Then, the fault characteristics and post-fault restoration of MVDC and MVAC distribution systems are compared and investigated through case studies. Time-domain simulations have been conducted in PSCAD/EMTDC. The work in this paper will be valuable for the protection design for future hybrid MV AC/DC systems.
\end{abstract}

Keywords-DC fault; DC protection; DC post-fault restoration; MVDC; $A C$ fault; $A C$ post-fault restoration; $D C$ circuit breaker; $M V A C$.

\section{INTRODUCTION}

Power generation plants, transmission grids and distribution networks are constantly expanding to cope with growing demand. At the same time, the environmental impact due to the burning of fossil fuels needs to be minimized. Renewable energy has a key role to play in this effort [1].

The fast development of power electronics converter based direct-current (DC) technology is driving the transformation of power energy systems [2]-[3]. The DC technology is raising more and more attractions in the top and tail of energy networks: transmission and distribution electric power systems. It is because that the DC infrastructure shows excellent performance in integrating renewable energy and providing affordable, reliable, and flexible power.

The voltage source converter (VSC) technology is becoming an attractive alternative to conventional line commutated converters (LCCs) for its features. These include [4]-[5]: 1) compact and flexible station layouts, with low space requirements, and a scalable system design; 2) a high dynamic performance and stable operation with AC

This work was supported by the Key Laboratory of Modern Power System Simulation and Control \& Renewable Energy Technology, Ministry of Education of China and the National Natural Science Foundation of China (51807102). networks; 3) capability of supplying power to passive networks and black-start; 4) an independent control of active and reactive power; and 5) no voltage polarity reversal during power flow reversal. Therefore, VSC-based DC systems are more suitable for distributed renewable energy integration than their LCC counterpart.

The feasibility, operation and control, and protection of building VSC-based MVDC grids and hybrid MV AC/DC distribution networks have been studied in the literature [2][3], [6]-[13]. The real applications of MVDC technology in the ANGLE-DC and Tangjiawan projects have been presented in [6]-[7]. The power-voltage coordinated control of using the VSCs in hybrid AC/DC distribution networks have been proposed in [2]-[3]. References [8]-[9] investigate the fault protection of AC grid-side unbalanced fault in a distribution network with a soft-open-point. The feasibility of converting the existing AC distribution network into DC operation has been studied in [10]-[12]. In [13], the analysis of harmonic transfer through an MVDC link has been conducted.

Although VSC-based MVDC grids complement the existing MVAC grids by making them more functional and controllable, their large-scale development still faces a number of challenges. One of the major obstacles is DC fault protections [14]. Effective DC protection methods are needed to minimize the impact from DC faults and ensure the secure operation of DC systems. There are three possible solutions to clear dc faults within a DC network: using AC circuit-breakers (ACCBs); using DC circuit breakers (DCCBs) and using fault blocking converters. The application issues associated with the above protection strategies in MVDC grids need to be investigated. For instance, the impact of DCCB operation on MVDC systems and the post-fault restoration of MVDC systems. Moreover, the comparison of the fault protection and post-fault restoration of MVDC and MVAC systems should be investigated to provide guidance for mitigating negative impacts on system operation and control.

In this paper, possible solutions for protecting DC faults in MVDC systems are first introduced. Then, fault 
characteristics and post-fault restoration of MVAC and MVDC distribution systems are compared and investigated through simulations conducted in PSCAD/EMTDC.

\section{FAUlt PROTECTION METHODS FOR MVDC SYSTEMS}

The fault detection, isolation and restoration have been widely studied in the literature [15]-[16]. Therefore, only protection methods for DC systems are discussed in this section.

\section{A. Using AC Circuit Breakers}

There are ACCBs and normally closed sectionalizing switches in AC distribution networks [15], [17]. An AC fault will be firstly detected and located. Then, ACCBs will open to isolate the fault. These processes can be tens to hundreds of milliseconds in distribution networks [9], [17]. Finally, the system will be restored to re-power the loads beyond the faulted zone.

For DC systems, ACCBs are already equipped on the $\mathrm{AC}$ side of converters and can be one option to protect the DC network, as shown in Fig. 1. The so-called "Handshaking" method which employs ACCBs and fast DC switches has been proposed in [18]. In order to extinguish the DC fault currents, all converters need to be blocked once a DC fault is detected. Then ACCBs will be tripped. Fast DC switches are available at both ends of each line and are used to isolate the faulted line under zero current. The healthy circuits will be restored once the faulted line is isolated. A progressive fault isolation and grid restoration strategy using ACCBs are proposed in [19]. The strategy reduces the grid outage time through the progressive restoration of the DC grid.

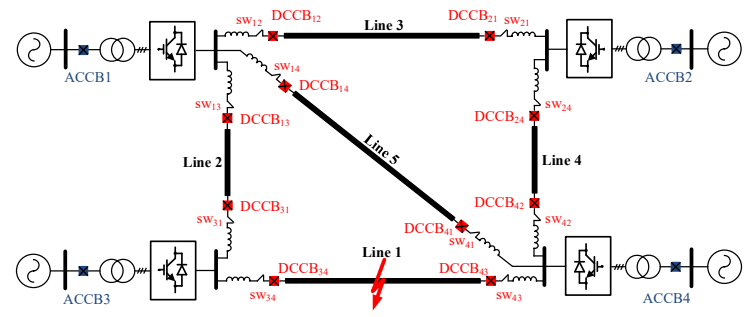

Fig. 1. Schematic diagram of a 4-terminal DC grid equipped with ACCBs, DCCBs and fast DC switches.

The advantages of using ACCBs are that it is simple and economic. This approach greatly reduces the investment cost of building a large-scale multi-terminal DC grid. However, the long fault isolation time (several hundred milliseconds or longer) associated with this strategy will lead to the deenergization of the entire DC grid [18]-[19]. This, in turn, may negatively affect the system secure operation.

\section{B. Using DC Circuit Breakers}

The absence of current zero-crossings in a DC system inherently makes DC current interrupting more difficult than that in AC systems. DC fault currents need to be isolated within several milliseconds. Even though some manufacturers have proposed several fast DCCBs, their high capital costs limit their applications [20]-[21]. Therefore, fast and economical DC protection is one of the main obstacles for the deployment of large-scale DC networks. Fig. 1 shows the application of DCCBs in a DC grid. Normally, DCCBs are installed at the two ends of a DC line. Sometimes, a single DCCB is needed to protect a DC bus.

The protection and post-fault restoration of DC networks have been widely studied in the literature. In a DC grid using DCCBs to provide fast clearance of a DC fault, two main solutions appear: one is to apply the same protection philosophy and principles used as AC systems which are called as the "Fully Selective Approach" [22]-[23] and the other is the "Open Grid" concept [24]-[25].

\section{Using Fault Blocking Converters}

The converters, such as full-bridge modular multi-level converter (FB-MMC) [26], clamp-double MMC (CD MMC) [27] and alternate arm converter (AAC) [28], have the capability of blocking AC infeeding currents by blocking the converters [29]. Once all of the converters within the DC grid are blocked, DC fault currents will start to naturally decay to zero so that fast DC switches are able to operate to isolate the faulted circuits. The converters will be de-blocked once the faulted line is isolated and the DC grid will start to restore to a new steady state.

The higher capital costs and power losses of using fault blocking converters are the main drawbacks of employing this technology in real applications. This may become even worse in medium or low voltage systems. Moreover, although the fault can be isolated without using DCCBs, the whole DC network will suffer an interruption of service and its associated AC systems will be impacted as well.

\section{Comparison of MVAC and MVDC System Protection and Restoration}

The comparison of MVAC and MVDC system protection and restoration are given in Table I. It should be mentioned that, due to the low impedance of DC circuits, a DC fault will propagate to all the converters at almost the same time [24]. All the VSC connected AC systems will be affected. From the viewpoint of AC systems, a fault within a DC network can be seen as "multiple faults" which may exacerbate the negative impact of the fault [23].

TABLE I

COMPARISON OF AC AND DC SYSTEM PROTECTION AND RESTORATION

\begin{tabular}{lll}
\hline \hline Items & AC systems & DC systems \\
\hline $\begin{array}{l}\text { Fault interrupting } \\
\text { devices }\end{array}$ & ACCB & $\begin{array}{l}\text { DCCB, ACCB and } \\
\text { converters }\end{array}$ \\
\hline $\begin{array}{l}\text { Fault detection and } \\
\text { location time }\end{array}$ & $\begin{array}{l}\text { Tens to hundreds of } \\
\text { milliseconds }\end{array}$ & Several milliseconds \\
\hline Fault isolation time & $\begin{array}{l}\text { Tens to hundreds of } \\
\text { milliseconds }\end{array}$ & Several milliseconds \\
\hline $\begin{array}{l}\text { System restoration } \\
\text { devices }\end{array}$ & $\begin{array}{l}\text { ACCB, AC switches, } \\
\text { sectionalizing switches }\end{array}$ & $\begin{array}{l}\text { DCCB, DC switches, } \\
\text { ACCB and converters }\end{array}$ \\
\hline $\begin{array}{l}\text { System restoration } \\
\text { time }\end{array}$ & Seconds to minutes & $\begin{array}{l}\text { Tens to hundreds of } \\
\text { milliseconds }\end{array}$ \\
\hline \hline
\end{tabular}

As for AC networks, the system restoration may need help from the normal open ACCBs which will be studied in the following section. However, the closing of these normal open ACCBs may lead to transient overcurrents [10]. The black-start and static synchronous compensator operating capability of VSCs are helpful during the post-fault restoration processes. 

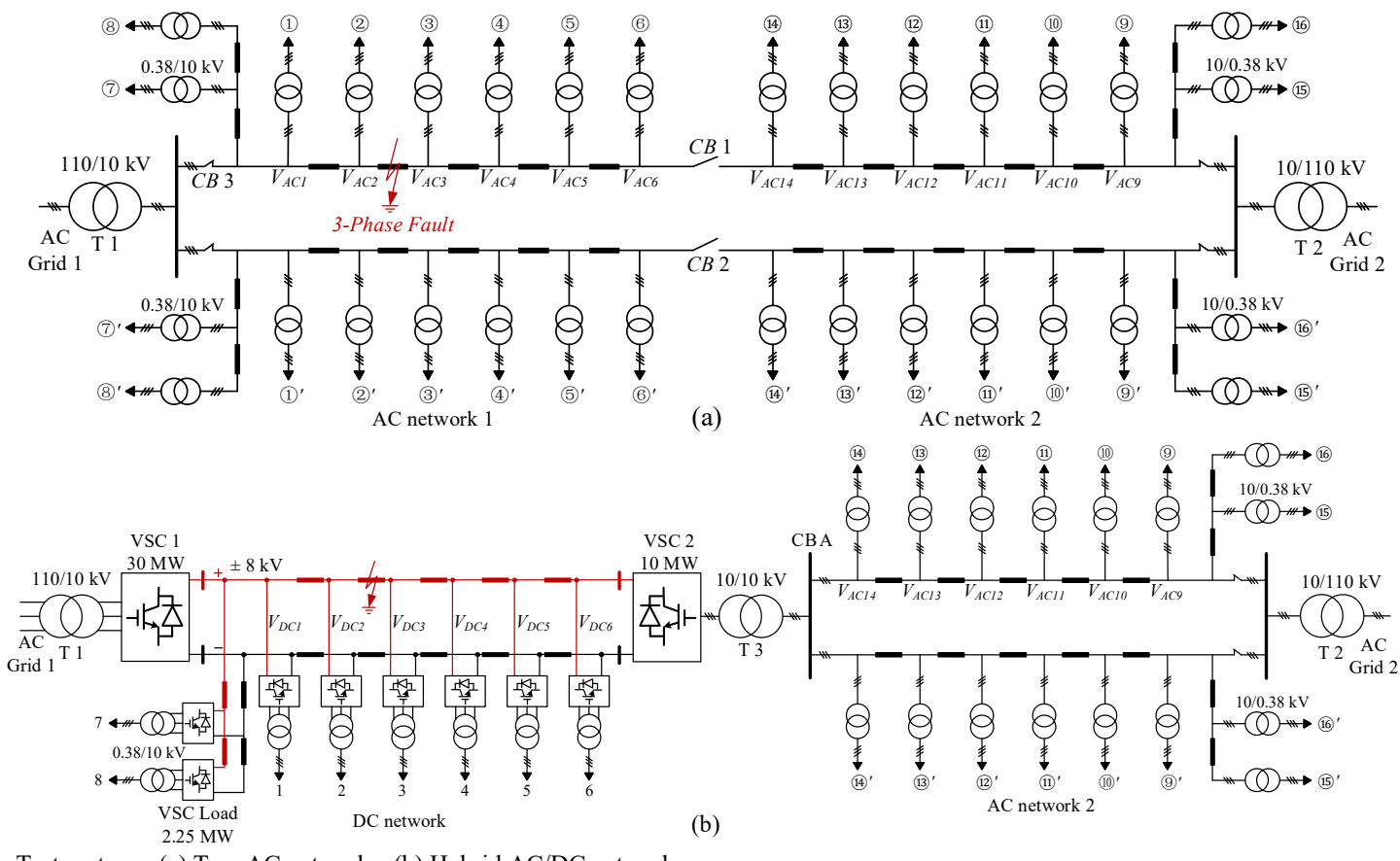

Fig. 2. Test systems. (a) Two AC networks; (b) Hybrid AC/DC network.

\section{CASE Studies AND ANALysis}

In this section, the modeling of the test systems and the operation and control sequences during AC and DC faults in the test systems will be presented. The systems are modeled in PSCAD/EMTDC. The test systems are depicted in Fig. 2.

\section{A. System Modelling}

Fig. 2(a) shows the configurations of a distribution system with two $10 \mathrm{kV}$ MVAC networks. The two networks are identical. Their main $\mathrm{AC}$ grids are at $110 \mathrm{kV}$. The loads are supplied through $10 \mathrm{kV}$ double-circuit overhead lines (OHLs). For instance, the loads (1) and (1)' are at one load point but equally supplied by the double circuits. The length of each segment is $1 \mathrm{~km}$. The loads are modelled as fixed loads with the capacity of $P+\mathrm{j} Q$. The parameters of the MVAC networks are given in Table II.

TABLE II

PARAMETERS OF THE MVAC NETWORK

\begin{tabular}{ll}
\hline \hline Parameters & Values \\
\hline AC grid frequency $(\mathrm{Hz})$ & 50 \\
Main grid voltage $(\mathrm{kV})$ & 110 \\
Main grid transformer capacity $(\mathrm{MVA})$ & 20 \\
Main grid transformer ratio $(\mathrm{kV} / \mathrm{kV})$ & $110 / 10$ \\
Main grid transformer leakage reactance (p.u.) & 0.1 \\
Load transformer capacity $(\mathrm{MVA})$ & 1.2 \\
Load transformer ratio $(\mathrm{kV} / \mathrm{kV})$ & $10 / 0.38$ \\
Load transformer leakage reactance (p.u.) & 0.1 \\
Resistance of the OHL $(\Omega / \mathrm{km})$ & 0.13 \\
Inductance of the $\mathrm{OHL}(\mathrm{H} / \mathrm{km})$ & 0.0127 \\
Load at each load point $(\mathrm{MW}+\mathrm{jMVar})$ & $2+\mathrm{j} 0.4$ \\
\hline \hline
\end{tabular}

The two AC networks can be connected through the two ACCBs (CBs 1 and 2) which are normally opened in normal operations. The CBs 1 and 2 can be closed for system reconfigurations and post-fault restorations.

Fig. 2(b) shows the configuration of the hybrid MV AC/DC network. The MVAC network 1 are replaced by \pm

$8 \mathrm{kV}$ MVDC link. It can be seen that there are only two conductors used as the positive and negative poles. Therefore, the capital cost of the lines is lower than its AC counterpart. However, two VSC stations are needed to be built at the two ends of the circuits which are the main cost of the MVDC system. The AC loads in this system will be supplied through DC/AC converters which are VSCs in this study. As the capacity of DC supply is higher than AC supply, each load can be supplied by a single converter instead of being supplied by the double-circuit in the AC system. The high capital cost and power losses of the two main VSCs and the load VSCs are the main drawbacks of utilizing DC technologies. However, the power capacity of the MVDC link can be much higher than the MVAC link which may be more cost-effective in the long-term operation.

As shown in Fig. 2(b), the DC network is connected to the AC network 2 via VSC 2's converter transformer. As the studied system is an MVDC system, MMCs may be not suitable due to their higher cost and power losses compared to two-level VSCs. Therefore, VSCs 1 and 2 and all of the load VSCs are all modelled using two-level VSCs. The DC side of the VSCs is solidly grounded. The VSC 1 controls the DC voltage and reactive power. The VSC 2 controls the active and reactive power. The load VSCs operate at active power and $\mathrm{AC}$ voltage control mode and they will be blocked and re-started based on their local protections quickly. The parameters of the converters are given in Table III.

\section{B. Dynamic Performances}

Before studying the fault characteristics of the $\mathrm{AC}$ and DC systems, the steady-state and dynamic performances will be studied first. Fig. 3 illustrates the performance of the two systems in Fig. 2. The locations of the voltage measurements $\left(\mathrm{V}_{\mathrm{ACx}}\right.$ and $\left.\mathrm{V}_{\mathrm{DCx}}\right)$ are shown in Fig. 2. 
TABLE III

PARAMETERS OF THE VSCS

\section{VSC 1}

\begin{tabular}{lc}
\hline Capacity (MW) & 30 \\
Interface transformer capacity (MVA) & 33 \\
Interface transformer ratio $(\mathrm{kV} / \mathrm{kV})$ & $110 / 10$ \\
Interface transformer leakage reactance (p.u.) & 0.12 \\
DC capacitor $(\mu \mathrm{F})$ & 5000 \\
DC terminal inductor (mH) & 0.003 \\
\hline \multicolumn{1}{c}{ VSC 2 } \\
\hline Capacity (MW) \\
Interface transformer capacity (MVA) \\
Interface transformer ratio $(\mathrm{kV} / \mathrm{kV})$ & 10 \\
Interface transformer leakage reactance (p.u.) & $10 / 10$ \\
DC capacitor $(\mu \mathrm{F})$ & 0.12 \\
DC terminal inductor (mH) & 4000 \\
\hline \multicolumn{1}{c}{ Load VSCs } & 0.0025 \\
\hline Capacity (MW) & 2 \\
Interface transformer capacity $(\mathrm{MVA})$ & 2.2 \\
Interface transformer ratio $(\mathrm{kV} / \mathrm{kV})$ & $10 / 0.38$ \\
Interface transformer leakage reactance (p.u.) & 0.1 \\
DC capacitor $(\mu \mathrm{F})$ & 500 \\
DC terminal inductor $L_{\mathrm{dc}}(\mathrm{mH})$ & 0.002 \\
\hline \hline
\end{tabular}

Fig. 3(a) shows that the voltages along the distribution line are different due to the voltage drops. The voltage $\mathrm{V}_{\mathrm{AC} 6}$ at the end of the line drops to 0.955 p.u.. As the two networks are identical, the steady-state voltages shown in Fig. 3(b) are the same as Fig. 3(a). To increase the voltage $\mathrm{V}_{\mathrm{AC1} 14}$ at the end of network 2 to 0.98 p.u., the $10 \mathrm{kV}$ side tap changer of the transformer T2 in Fig. 2(a) is changed to 1.03 p.u. at $\mathrm{t}=0.75$ $\mathrm{s}$ and is changed back to 1.00 p.u. at $\mathrm{t}=1.00 \mathrm{~s}$. Fig. $3(\mathrm{~b})$ shows that all voltages along the distribution line are increased during this period. As the ACCBs 1 and 2 are open, the network 1 is not affected. It should be noted that increasing the line voltage by changing the tap changer may lead to the suffering of overvoltage at points close to the transformer.
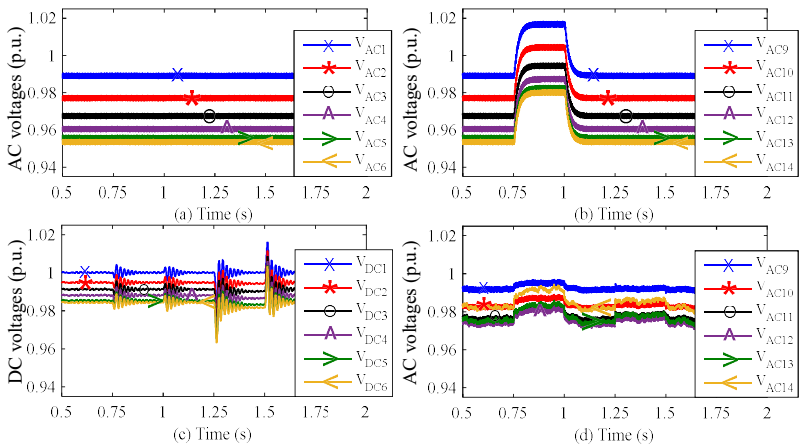

Fig. 3. System responses in the. (a) Voltages at the AC Network 1 in Fig. 2(a); (b) Voltages at the AC Network 2 in Fig. 2(a); (c) Converter terminal voltages at the DC Network in Fig. 2(b); (d) Voltages at the AC Network 2 in Fig. 2(b).

Fig. 3(c) shows the DC terminal voltages of the converters in the MVDC network. The voltages are all above 0.985 p.u.. VSC 2 injects $3 \mathrm{MW}$ and $0.6 \mathrm{MVar}$ to the AC network 2. Due to the support from VSC 2, the voltages at the points close to VSC 2 are improved to above 0.97 p.u. as shown in Fig. 3(d). Especially, the voltage $\mathrm{V}_{\mathrm{AC} 14}$ has been increased to 0.983 p.u., as it is closely connected to VSC 2. The reactive power of VSC 2 is increased to 1.2 MVar at $\mathrm{t}=$ $0.75 \mathrm{~s}$ and changed back to $0.6 \mathrm{MV}$ ar at $\mathrm{t}=1.00 \mathrm{~s}$. As shown in Fig. 3(d), during this period, the voltages at AC network 2 are all increased without a large increase of the voltages of the points far away from the VSC 2. The dynamic process of changing the reactive power leads to small oscillations of the DC voltages which become stable within $0.1 \mathrm{~s}$. The active power of VSC 2 is increased to $4.5 \mathrm{MW}$ at $t=1.25 \mathrm{~s}$ and is changed back to $3 \mathrm{MW}$ at $t=1.50 \mathrm{~s}$. During this period, the voltages at $\mathrm{AC}$ network 2 are all increased, however, the increases are less than changing reactive power. Moreover, the oscillations of the DC voltages are larger than changing reactive power. However, the DC voltages also become stable within $0.1 \mathrm{~s}$.

The studies in this section show that the support from VSC can effectively improve the voltage profiles of its connected AC network. This can reduce the use of online tap changer of the transformer.

\section{System Performances against Faults}

In this section, the performances of the MVAC network and hybrid MV AC/DC network subject to faults are investigated. A permanent three-phase-to-ground $\mathrm{AC}$ fault is set in the middle of the line between loads (2) and (3) in the system shown in Fig. 2(a). A permanent positive pole-toground DC fault is set in the middle of the line between loads 2 and 3 in the system shown in Fig. 2(b).

Fig. 4 illustrates the fault responses and post-fault processes of the two cases. The fault occurs at $t=t_{1}$. Fig. 4(a) shows that the voltages $\mathrm{V}_{\mathrm{AC} 1}$ and $\mathrm{V}_{\mathrm{AC} 2}$ drop to 0.55 p.u. and 0.18 p.u. while the voltages at other points drop to zero. The main breaker CB 3 is tripped at $t=t_{2}$ to isolate the fault. The interval between $t_{1}$ and $t_{2}$ is used for fault detection, location and isolation which can be hundreds of milliseconds [9],[11]. The AC fault currents through the CB 3 reach to 8.71 p.u. in this period. The fault is cleared, and the faulted line is fixed at $t=t_{3}$. The interval between $t_{2}$ and $t_{3}$ can last from minutes to hours. The CB 3 is reclosed at $t=t_{4}$ then the loads on this line are powered again through its main grid.
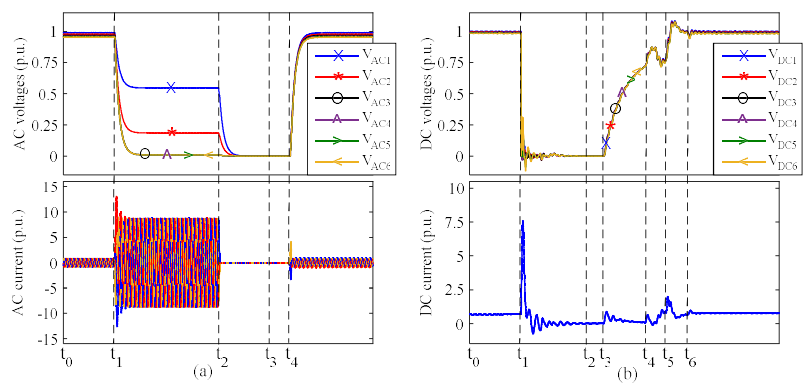

Fig. 4. Fault responses and post-fault restorations processes of the AC network 1 and the DC network. (a) Voltages and currents of the AC network 1; (b) Voltage and currents of the DC network.

Fig. 4(b) shows the DC voltages and current during the DC fault. In this case, the ACCB based protection scheme is employed [18]. This ACCB based protection scheme has been applied in the ANGLE-DC project. The converters will be blocked immediately either the current is 2 p.u. of its rated current or the DC terminal voltage drop to lower than 0.80 p.u. of its rated voltage. Then, the converter AC side breakers will be tripped to isolate the fault. It can be seen that the voltages along the DC lines drop to zero immediately after the fault and the DC fault current at the terminal of the VSC 
1 reach to 7.52 p.u. within $8 \mathrm{~ms}$. The fault is cleared, and the line is fixed at $t=t_{2}$. As most of the distribution networks are not fully automatic, this process may need human operators [16]. Thus, the interval between $t_{1}$ and $t_{2}$ can last from minutes to hours as well. The DC system starts to restore at $t$ $=\mathrm{t}_{3}$. To limit the current during the startup process, the VSC 1 is charged through a startup resistor which is bypassed at $t$ $=\mathrm{t}_{4}$. The VSC 1 is deblocked at $\mathrm{t}=\mathrm{t}_{5}$. Then the DC voltage is regulated to its rated value. The $\mathrm{AC}$ side circuit breaker of VSC 2 is reclosed $t=t_{6}$. Then, the VSC 2 and load converters are deblocked and the system is restored.

It should be mentioned that the AC network 2 is affected by the DC fault due to the connection with VSC 2. Fig. 5 illustrates the voltages in the AC network 2. $\mathrm{V}_{\mathrm{AC} 14}$ drops to 0.83 p.u. and $\mathrm{V}_{\mathrm{AC}}$ drops to less than 0.95 p.u.. This may trigger the relay protection of the AC network 2 and therefore exacerbate the fault. The whole system protection design should consider this kind of mutual interactions between AC and DC systems.

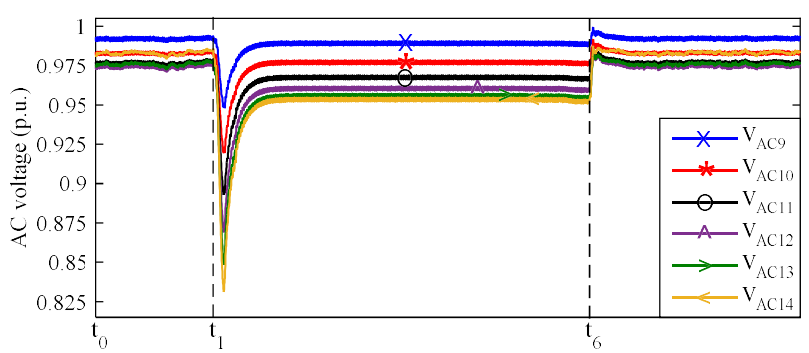

Fig. 5. Voltage profiles of the AC network 2 during and after the DC fault.

The use of DCCBs can reduce the negative impact of DC faults on system operation. There have been many DCCBs for MVDC applications [20]. Fig. 6 illustrates the system responses of using DCCBs to protect the DC network. DCCBs are deployed at the DC terminals of VSCs 1 and 2. In this study, a $5 \mathrm{~ms}$ delay after the fault is used to emulate the operating time of DCCBs. Due to the fast operating speed, the VSC 1's DC voltage of the positive pole just drops to 0.99 p.u. and the DC fault current is interrupted at 1.96 p.u.. The system can use the residual voltage to achieve a fast system restoration, for instance in several seconds. As the fault is isolated quickly, the AC network 2 is less affected compared to the case of using ACCBs.

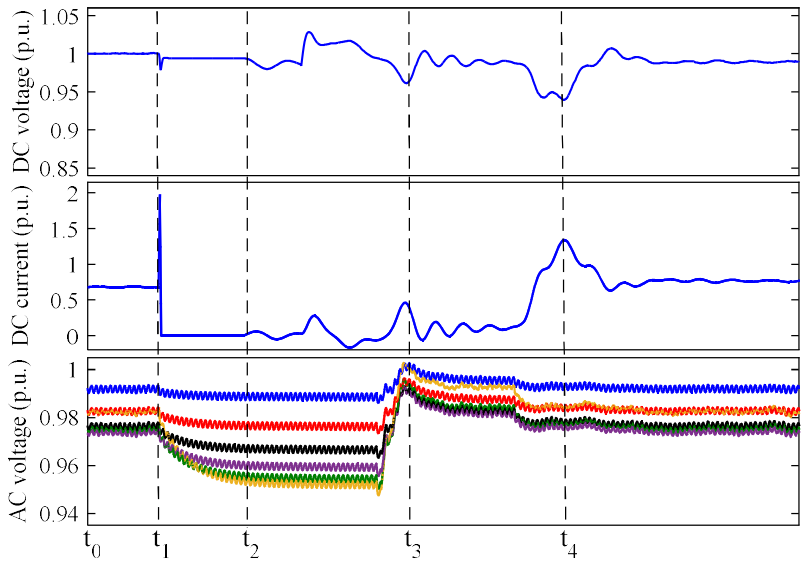

Fig. 6. System responses when the DCCBs are used.
It should be mentioned that the MVDC network uses a monopolar configuration. Any kinds of DC faults, pole-topole or pole-to-ground, will lead to the outage of the whole DC system. However, for the MVAC system, the load in one circuit can be relocated to other circuits through network reconfiguration. Take the above case for example, after isolating the faulted line using the sectionalizing switches, the loads (1), (2), (7) and (8) can be re-powered by closing the CB 3 at $t=t_{1}$ as shown in Fig. 7. In order to supply the loads on the outage line, the voltage is increased by changing the tap changer in the AC network 2 at $\mathrm{t}=\mathrm{t}_{2}$. The loads (4)-(6) are resupplied when the CB 1 is closed. Load (3) is not connected because it will lower the voltages to less than 0.93 p.u.. Similarly, if there is a fault occurs in the main grid of the AC network 1, part of the loads can be supplied by the $\mathrm{AC}$ network 2 through network reconfiguration.

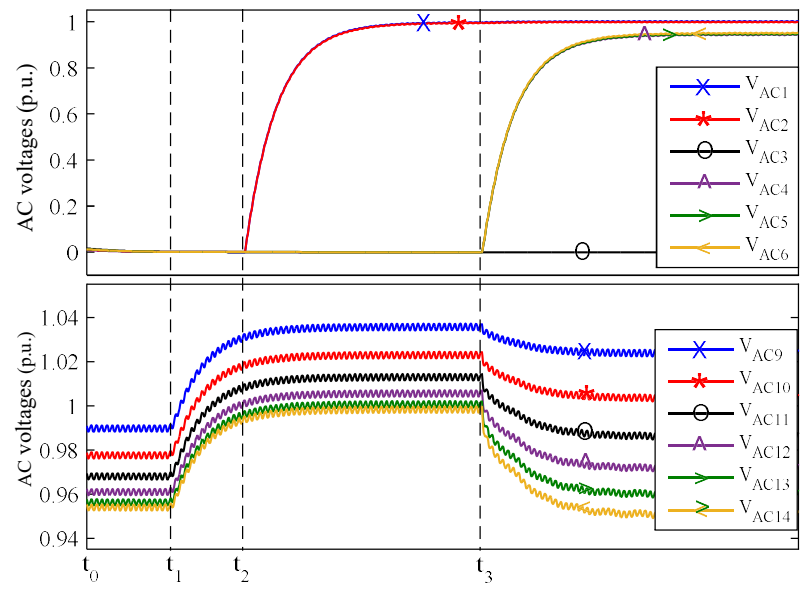

Fig. 7. Voltage profiles of the two AC networks during the network reconfiguration.

It should be mentioned that the bipolar configuration which employs two independently controlled asymmetrical monopolar VSCs can achieve 50\% power transmission during a fault in one pole [30]-[31]. This improves the system reliability, however, it will double the capital costs and therefore may not be economical for MV applications.

\section{CONCLUSION}

In this paper, the fault protection approaches for $\mathrm{AC}$ and DC systems have been investigated. The challenges of protecting DC grids from DC faults are first presented. The comparison of the fault protection and post-fault restoration of AC and DC system has been conducted. Time-domain simulations in PSCAD/EMTDC have been carried out to investigate the dynamic and fault behaviors of the $\mathrm{AC}$ and DC systems. Both ACCB and DCCB based protection methods have been applied in the test systems. The studies show that the application of a DC link can effectively improve the voltage profile and control flexibility of an $\mathrm{AC}$ network. Moreover, the response time of DC systems during a fault and the post-fault restoration time is much faster than AC systems. However, it should be mentioned that the adopted protection strategies for a hybrid MV AC/DC system must consider the practical requirement on the reliability of power supply and capital costs. Moreover, the whole system 
protection design of a hybrid $\mathrm{AC} / \mathrm{DC}$ system needs to consider the interactions between the $\mathrm{AC}$ and $\mathrm{DC}$ systems during faults.

\section{REFERENCES}

[1] G. Li, "Analysis and protection of HVDC systems subject to ac and dc faults," PhD thesis, Cardiff University, Mar. 2018.

[2] L. Zhang, et al., "A medium voltage hybrid AC/DC distribution network and its economic evaluation," in 12th IET International Conference on $A C$ and DC Power Transmission (ACDC 2016), Beijing, 2016, pp. 1-6.

[3] L. Zhang, et al., "Power-voltage coordinated control in hybrid AC/DC medium voltage distribution networks based on VSC," Proceedings of the CSEE, vol. 36, no. 22, pp. 6067-6075, November 2016.

[4] G. Li, C. Li and Dirk van Hertem, "HVDC technology overview," HVDC Grids: For Offshore and Supergrid of the Future, Wiley-IEEE Press, Chapter 3, pp.45-76, 2016.

[5] G. Li, et al., "Feasibility and reliability analysis of LCC DC grids and LCC/VSC hybrid DC grids," IEEE Access. vol. 7, pp. 22445-22456, 2019.

[6] B. Zhao, R. Zeng, Q. Song, Z. Yu and L. Qu, "Medium-voltage DC power distribution technology," The Energy Internet, Chapter 6, Woodhead Publishing, pp. 123-152, 2019.

[7] T. Joseph, J. Liang, G. Li, A. Moon, K. Smith and J. Yu, "Dynamic control of MVDC link embedded in distribution network: - Case study on ANGLE-DC," in 2017 IEEE Conference on Energy Internet and Energy System Integration (EI2), Beijing, 2017, pp. 1-6.

[8] A. Aithal, G. Li and J. Wu, "Grid Side Unbalanced Fault Detection using Soft Open Point in an Electrical Distribution Network." Energy Procedia, vol. 105, pp. 2859-2864, May 2017.

[9] A. Aithal, G. Li and J. Wu, "Performance of an electrical distribution network with Soft Open Point during a grid side AC fault." Applied Energy, vol. 227, pp. 262-272, Oct. 2017.

[10] L. Zhang, J. Liang, W. Tang, G. Li, Y. Cai and W. Sheng, "Converting $\mathrm{AC}$ Converting $\mathrm{AC}$ distribution lines to DC to increase transfer capacities and DG penetration," IEEE Trans. Smart Grid, vol. 10, no. 2, pp. 1477-1487, March 2019.

[11] A. Shekhar, L. M. Ramirez-Elizondo, T. B. Soeiro and P. Bauer, "Boundaries of Operation for Refurbished Parallel AC-DC Reconfigurable Links in Distribution Grids," IEEE Trans. Power Del., 2018 (Early access).

[12] A. Shekhar, E. Kontos, L. R. Elizondo, A. R. Mor, P. Bauer, "Grid capacity and efficiency enhancement by operating medium voltage AC cables as DC links with modular multilevel converters," International Journal of Electrical Power \& Energy Systems, vol. 93, pp. 479-493, Dec. 2017.

[13] T. Joseph et al., "Analysis of harmonic transfer through an MVDC link," in 15th IET International Conference on $A C$ and DC Power Transmission (ACDC 2019), Coventry, UK, 2019, pp. 1-6.

[14] M. Monadi, C. Koch-Ciobotaru, A. Luna, J. Ignacio Candela and P. Rodriguez, "Multi-terminal medium voltage DC grids fault location and isolation," IET Generation, Transmission \& Distribution, vol. 10, no. 14, pp. 3517-3528, 4112016.

[15] A. Zidan et al., "Fault Detection, Isolation, and Service Restoration in Distribution Systems: State-of-the-Art and Future Trends," IEEE Trans. Smart Grid, vol. 8, no. 5, pp. 2170-2185, Sept. 2017.

[16] S. S. Gururajapathy, H. Mokhlis and H. A. Illias, "Fault location and detection techniques in power distribution systems with distributed generation: A review," Renewable and Sustainable Energy Reviews, vol. 74, pp. 949-958, Jul. 2017.

[17] B. Li and H. Liu, "Current limiting methods for VSC-based DC distribution systems," Energy Procedia, vol. 147, pp. 2257-2263, 2017.

[18] L. Tang and B. T. Ooi, "Locating and Isolating DC Faults in MultiTerminal DC Systems," IEEE Trans. Power Del., vol. 22, no. 3, pp. 1877-1884, July 2007.

[19] R. Dantas, J. Liang, C. E. Ugalde-Loo, A. Adamczyk, C. Barker and R. Whitehouse, "Progressive Fault Isolation and Grid Restoration Strategy for MTDC Networks," IEEE Trans. Power Del., vol. PP, no. 99, pp. 1-1.
[20] G. Li, et al., "Frontiers of DC circuit breakers in HVDC and MVDC systems," in 2017 IEEE Conference on Energy Internet and Energy System Integration (EI2), Beijing, 2017, pp. 1-6.

[21] S. Wang, et al., "Coordination of MMCs with Hybrid DC Circuit Breakers for HVDC Grid Protection," IEEE Trans. Power Del. vol. 34, no. 1, pp. 11-22, Feb. 2019.

[22] B. Geebelen, W. Leterme and D. V. Hertem, "Analysis of DC breaker requirements for different HVDC grid protection schemes," in $A C$ and DC Power Transmission, 11th IET International Conference on, Birmingham, 2015, pp. 1-7.

[23] G. Li, J. Liang, C. E. Ugalde-Loo, P. Coventry and J. Rimez, "Dynamic interactions of DC and AC grids subject to DC faults," in 2016 IEEE 8th International Power Electronics and Motion Control Conference (IPEMC-ECCE Asia), Hefei, 2016, pp. 2627-2633.

[24] C. D. Barker and R. S. Whitehouse, "An alternative approach to HVDC grid protection," in $A C$ and DC Power Transmission (ACDC 2012), 10th IET International Conference on, vol., no., pp.1-6, 4-5 Dec. 2012.

[25] GE Think Grid, Implementing the protection and control of future HVDC grids. [Online]. Available: http://www.thinkgrid.org/implementing-protection-and-control-future-hvdc-grids (Last access: 21-05-2019)

[26] A. Nami, J. Liang, F. Dijkhuizen, and G. D. Demetriades, "Modular multilevel converters for HVDC applications: Review on converter cells and functionalities," IEEE Trans. Power Electron., vol. 30, no. 1, pp. 18-36, Jan. 2015.

[27] R. Marquardt, "Modular multilevel converter: A universal concept for HVDC-networks and extended DC bus-applications," in Proc. Int. Power Electron. Conf., 2010, pp. 502-507.

[28] M. M. C. Merlin, et al., "The Alternate Arm Converter: A New Hybrid Multilevel Converter With DC-Fault Blocking Capability," IEEE Trans. Power Del., vol. 29, no. 1, pp. 310-317, Feb. 2014.

[29] C. Liu et al., "An Isolated Modular Multilevel Converter (I-M\$2\$C) Topology based on High-Frequency Link (HFL) Concept," IEEE Trans. Power Electron. Jun. 2019. (Early Access)

[30] G. Li, J. Liang, F. Ma, C. E. Ugalde-Loo and H. Liang, "Analysis of single-phase-to-ground faults at the valve-side of HB-MMCs in HVDC systems," IEEE Trans. Ind. Electron., vol. 66, no. 3, pp. 2444-2453, March 2019.

[31] G. Li, W. Liu, T. Joseph, J. Liang and Z. Song, "Double-Thyristor based Protection for Valve-Side Single-Phase-to-Ground Faults in HB-MMC based Bipolar HVDC Systems," IEEE Trans. Ind. Electron., Jul. 2019. (Early Access) 